\title{
gु
}

\section{Size-Dependent Localization in Polydisperse Colloidal Glasses}

\author{
D. Heckendorf, ${ }^{1}$ K. J. Mutch, ${ }^{1}$ S. U. Egelhaaf, ${ }^{1}$ and M. Laurati ${ }^{1,2}$ \\ ${ }^{1}$ Condensed Matter Physics Laboratory, Heinrich Heine University, Universitätsstrasse 1, 40225 Düsseldorf, Germany \\ ${ }^{2}$ División de Ciencias e Ingenierías, Universidad de Guanajuato, Loma del Bosque 103, 37150 León, Mexico
}

(Received 13 March 2017; published 28 July 2017)

\begin{abstract}
We have investigated concentrated suspensions of polydisperse hard spheres and have determined the dynamics and sizes of individual particles using confocal microscopy. With increasing concentration, the dynamics of the small and large particles start to differ. The large particles exhibit slower dynamics and stronger localization. Moreover, as the particle size increases, the local volume fraction $\phi_{\text {loc }}$ also increases. In the glass state, the localization length significantly decreases beyond $\phi_{\text {loc }} \approx 0.67$. This suggests a link between local crowding and dynamical heterogeneities. However dynamical arrest of subpopulations seems not directly linked to a large value of $\phi_{\text {loc }}$, indicating the importance of collective effects.
\end{abstract}

DOI: 10.1103/PhysRevLett.119.048003

Colloidal dispersions are applied in various fields, from paints to food stuff and medicines [1,2]. Industrial dispersions typically contain particles with a broad size distribution; i.e., they are polydisperse. Polydispersity can severely affect the behavior of colloidal dispersions. For example, if monodisperse particles crystallize they form one solid phase whereas in moderately polydisperse samples fractionation into multiple coexisting solid states can occur [3-11] and at larger polydispersities crystallization is suppressed [12-14]. Crystallization can also be precluded by nonequilibrium effects, namely, the formation of glasses at volume fractions $\phi>\phi_{\mathrm{g}} \approx 0.58$ [15-23]. The glass state is linked to dynamical arrest caused by particles being trapped in cages formed by their nearest neighbors [23-28], at least until activated processes can restore longtime diffusion [29]. Polydispersity also affects glass formation. With increasing polydispersity the glass transition moves to larger $\phi[30,31]$. Similarly, random close packing (RCP) occurs at larger $\phi$ [32-35] because spheres of different sizes pack more efficiently. Using simulations based on realistic particle size distributions, the different sizes have been found to undergo separate glass transitions [30], which has also been predicted and observed for binary dispersions [36-41]. Thus, polydispersity has highly nontrivial effects on the glass transition and crystallization, but also on other phenomena, such as self-assembly [42], drying of colloidal dispersions [43], or friction [44].

Despite these clear and important effects of polydispersity, an experimental determination of the behavior of the different species in polydisperse samples is still lacking. This is due to experimental limitations. The size of individual particles or the particle size distribution can be determined quantitatively, for example by electron microscopy, analytical ultracentrifugation, or light scattering $[30,45,46]$. These techniques, however, do not yield the dynamics on an individual particle level. On the other hand, measurement techniques such as confocal microscopy can provide access to single-particle properties, e.g., the singleparticle dynamics $[21,22,24,47-49]$. The size of individual particles only became accessible by confocal microscopy with sufficient accuracy very recently $[6,19]$. This meant that the particle size and dynamics could not experimentally be determined on an individual particle level simultaneously. They could therefore not be linked and only ensemble-averaged parameters were determined. While they characterize the average effects of polydispersity, they do not provide information on the specific behavior of the different subpopulations.

Here we apply a recently proposed method to determine the particle size using confocal microscopy [6]. This allows us to simultaneously determine the particle size and the single-particle dynamics as well as the local volume fraction and other structural parameters. The dynamics and arrangements of subpopulations of particles hence become accessible. Our experiments reveal that, beyond the glass transition, the large particles exhibit much slower dynamics and are considerably more localized than the small particles. These findings provide the first experimental evidence that, in polydisperse systems, the dynamics of the individual species differ significantly. Furthermore, the slower dynamics and stronger localization of the large particles correlate with a higher local volume fraction $\phi_{\text {loc }}$. Beyond a critical value $\phi_{\text {loc }} \approx 0.67$, slow dynamics and strong localization are observed. This suggests a connection between a structural feature, local crowding, quantified by $\phi_{\text {loc }}$, and dynamical heterogeneities, which have been related to glass formation $[20,24,25,50]$. However, a large local volume fraction of individual particles seems not directly linked to the dynamical arrest of the whole sample. This indicates that glass formation also requires collective effects which involve the interplay of groups of particles.

The samples were dispersions of polydisperse polymethylmethacrylate (PMMA) spheres fluorescently labeled with nitrobenzoxadiazole (NBD) in a density and almost 
refractive index matched mixture of cis-decalin and cycloheptylbromide with $4 \mathrm{mM}$ of tetrabutylammoniumchloride salt added; density matching and all measurements were performed at $T=23^{\circ} \mathrm{C}$. In this solvent mixture the particles show hard-sphere-like behavior [51]. Their average radius was $\langle R\rangle=0.72 \mu \mathrm{m}$, as determined by dynamic light scattering, with a polydispersity of $\sigma \approx 13 \%$. The most concentrated sample with a volume fraction $\phi=0.61$ was prepared by diluting a sediment which was obtained by centrifugation at a temperature $T=35^{\circ} \mathrm{C}$ that temporarily introduces a solvent-particle density difference. The other samples were prepared by successive dilution which ensures that their relative concentrations are very reliable. After dilution, the samples were thoroughly mixed in a shaker for at least one day and then filled in vials whose bottoms were replaced by cover slips [52]. The volume fractions of the samples, $\phi$, were determined based on the volume fraction of the sediment which was assumed to be the volume fraction of random-close-packed spheres with a polydispersity $\sigma=13 \%$, i.e., $\phi_{\mathrm{RCP}}=0.66$ [32-34]. These values of $\phi$ are consistent with $\phi=(4 \pi N / 3 v)\left\langle R^{3}\right\rangle$ with the number of particles $N$ in the observation volume $v$ and the third moment $\left\langle R^{3}\right\rangle$ as determined from the size distribution.

After a few days, the samples were imaged using a confocal microscope (Nikon A1R-MP with a Nikon Plan Apo $60 \times$ oil immersion objective with $\mathrm{NA}=1.40$ ). For each sample five independent measurement series, taken at different positions in the sample, were performed, each consisting of three steps to determine (i) the particle sizes and structural parameters, (ii) the single-particle dynamics, and (iii) the particle sizes and structural parameters again. The three steps involved (i) 10 stacks of $512 \times 512 \times 68$ voxels $\left(\approx 51 \times 51 \times 10 \mu \mathrm{m}^{3}\right)$ about $15 \mu \mathrm{m}$ from the cover slip with a stack containing about $10^{5}$ particles and being acquired every $2.3 \mathrm{~s}$, (ii) a series of 3000 slices taken at the mid vertical plane of the previous stacks and acquired every 0.02 to $3 \mathrm{~s}$ depending on the sample which yields about $10^{4}$ trajectories [53], and (iii) 10 stacks again as in (i). Particle locations and trajectories were extracted using standard routines [47] and were subsequently refined [52]. The particle radii were determined following Refs. [6,54].

Based on the individual particle sizes, the distributions of the particle radius $P(R)$ were calculated for all samples (Fig. 1). As expected, the distributions are comparable. The average particle size $\langle R\rangle \approx 0.73 \mu \mathrm{m}$ is in agreement with the value determined by light scattering. The normalized polydispersity, $\sigma$, is quantified by $\sigma=$ $\left(\left\langle R^{2}\right\rangle-\langle R\rangle^{2}\right)^{1 / 2} /\langle R\rangle$ with the averages $\left\langle R^{n}\right\rangle=\int d R R^{n} P(R)$ and found to be $\sigma \approx 13 \%$. In the following we consider the behavior of all particles as well as the subpopulations of the $20 \%$ smallest and 20\% largest particles, respectively. A fraction of $20 \%$ of all particles ensures good enough statistics while maintaining sufficient selectivity. The size ratio between the average radii of the subpopulations of the largest and smallest particles, respectively, is about 1.55.

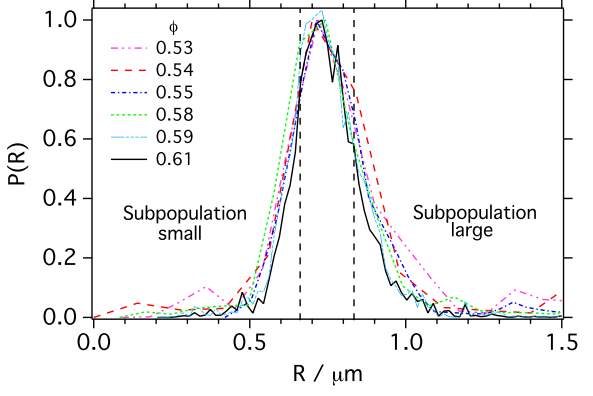

FIG. 1. Particle size distribution $P(R)$ for samples with different volume fractions $\phi$, as indicated. Dashed black lines delimit the subpopulations of the $20 \%$ smallest and $20 \%$ largest particles, respectively, for the sample with $\phi=0.61$

The particle dynamics are characterized by the mean squared displacement (MSD); $\left\langle\Delta r^{2}(t)\right\rangle=\left\langle\left(r_{i}\left(t, t_{0}\right)-\right.\right.$ $\left.\left.r_{i}\left(0, t_{0}\right)\right)^{2}\right\rangle_{t_{0}, i}$ where $t$ is the delay time, $t_{0}$ a time during the particle trajectory, and $\langle\cdots\rangle_{t_{0}, i}$ indicates an average over all times $t_{0}$ and either all particles $i$ in the observation volume or only a subpopulation thereof. Trajectories were corrected for any residual drift. With increasing delay time $t$, less trajectories are typically available. If at least $10^{3}$ independent trajectories were available at a particular delay time $t$, the MSD was considered reliable and is shown in Fig. 2. Since the MSDs of the subpopulations are based on significantly fewer particles, they are reliable up to shorter maximum delay times than the MSDs of all particles.

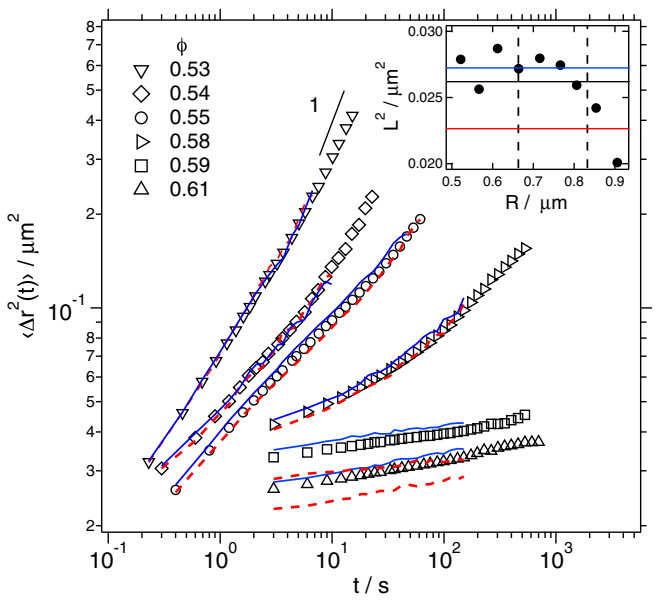

FIG. 2. Mean squared displacement (MSD), $\left\langle\Delta r^{2}(t)\right\rangle$, as a function of delay time $t$ for samples with different volume fractions $\phi$, as indicated. The MSDs are based on all particles (symbols), the 20\% smallest (blue solid lines) and the 20\% largest particles (red dashed lines). Inset: Squared localization length $L^{2}$, characterized by the MSD at the shortest observed delay time, $\left\langle\Delta r^{2}\left(t_{\mathrm{i}}=3 \mathrm{~s}\right)\right\rangle$, as a function of the particle radius $R$ for the sample with $\phi=0.61$. Vertical dashed lines indicate the $20 \%$ smallest and 20\% largest particles, respectively (as in Fig. 1), and the horizontal lines the mean localization lengths of the smallest, all, and the largest particles, respectively (top to bottom). 
The average MSDs show a progressive slowing down of the dynamics with increasing volume fraction $\phi$ (Fig. 2, symbols). At the highest $\phi \geq 0.59$, localization occurs with a very modest increase of the MSD that extends to long times $t$, in agreement with previous observations in similar polydisperse colloidal glasses [20,22,28]. This indicates the onset of dynamical arrest and glass formation. The average MSDs are compared to the MSDs of the small and large particles (Fig. 2, lines). At the smallest volume fractions, $\phi \leq 0.54$, the dynamics of the subpopulations are indistinguishable from the dynamics of all particles. For larger volume fractions, $0.55 \leq \phi \leq 0.58$, the dynamics start to differ with the large particles exhibiting slightly slower dynamics. The decoupling of the dynamics becomes pronounced for the arrested states with $0.59 \leq \phi \leq 0.61$. In particular, the large particles are significantly slower and more localized than the average particle, whereas the small particles show only slightly faster dynamics.

To investigate the size dependence in more detail, the size range $0.50 \mu \mathrm{m} \leq R \leq 0.95 \mu \mathrm{m}$ is divided into ten groups. For each group the MSD is determined at the shortest delay time observed for all samples, $t_{\mathrm{i}}=3 \mathrm{~s}$, which is taken to characterize the squared localization length, $L^{2}=\left\langle\Delta r^{2}\left(t_{i}\right)\right\rangle$. For $\phi=0.61$, the localization length is found to be about constant for the small and intermediate particles but decays strongly for the largest particles (Fig. 2, inset). Because of the small fraction of large particles (Fig. 1), the average localization length is similar to the localization length of the small and intermediate particles (Fig. 2, horizontal lines in the inset). Thus, although dynamical arrest is particularly strong for the large particles, they hardly contribute to the average behavior. For the other samples that show localization, $\phi=0.58$ and 0.59 , a less pronounced decay is observed while for the more dilute samples, $\phi \leq 0.55$, no significant dependence on $R$ is found [data presented later, Fig. 4(d)].

The information on the dynamics is complemented by structural information, namely, the pair distribution function $g(r)$. While $g(r)$ characterizes the distribution of all particles surrounding any particle, we also determined $g_{\mathrm{s}}(r)$ and $g_{1}(r)$ that characterize the distributions of all particles around a small and a large particle, respectively. All pair distribution functions show the characteristic features of concentrated colloidal suspensions (Fig. 3): a peak at a distance $r \approx 2\langle R\rangle$ that reflects the nearest neighbor shell formed by particles with slightly different sizes as well as less pronounced peaks that represent successive neighbor shells. These features are similar in $g(r), g_{\mathrm{s}}(r)$, and $g_{1}(r)$ and hence the structural arrangements around the small and large particles resemble those around any particle. Nevertheless, the first peak is moderately larger in the case of $g_{\mathrm{s}}(r)$ and especially in the case of $g_{\mathrm{l}}(r)$ than for $g(r)$. In both cases, the peaks are located slightly beyond $2\langle R\rangle$, which is expected in the case of large particles. However,

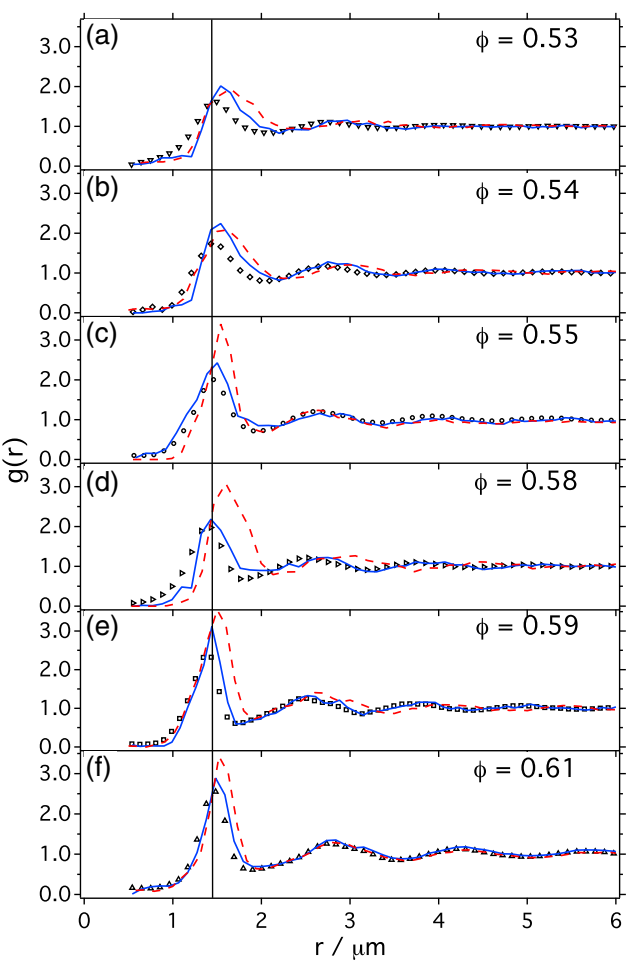

FIG. 3. Pair distribution functions for samples with different volume fractions (a) $\phi=0.53$, (b) 0.54 , (c) 0.55 , (d) 0.58 , (e) 0.59 and (f) 0.61 . They are based on the arrangement of all particles around any particle, $g(r)$ (symbols), around one of the $20 \%$ smallest particles, $g_{\mathrm{s}}(r)$ (blue solid lines), and around one of the $20 \%$ largest particles, $g_{1}(r)$ (red dashed lines). The vertical line indicates the average particle diameter $2\langle R\rangle$.

particles also tend to stay farther apart from small particles suggesting that small particles are less tightly packed. With increasing $\phi$, the heights of the peaks increase as the neighbor shells become more pronounced upon crowding, but there is no qualitative change in the particle arrangement visible, in contrast to the findings for the particle dynamics.

The different arrangements of the small and large particles are also seen in projections of a slice of the sample with $\phi=0.61$ [Fig. 4(a)]. The small particles are mostly isolated with only very few small particles arranged in short chainlike structures, consistent with the peak of $g_{\mathrm{s}}(r)$ being located beyond the particle diameter $2\langle R\rangle$. In contrast, the large particles are in close contact with their nearest neighbors and form large, compact and interconnected structures, as suggested by $g_{1}(r)$. Correspondingly and as indicated by the different areas under the first peaks of the pair distribution functions, the mean number of neighbors is higher for the large particles than for the small particles (data not shown).

The space available to a particle can be characterized by its Voronoi volume $V$ [indicated by the colors of the particles in Fig. 4(a)], where the radical Voronoi construction is used to account for the different particle radii [55]. 
(a)
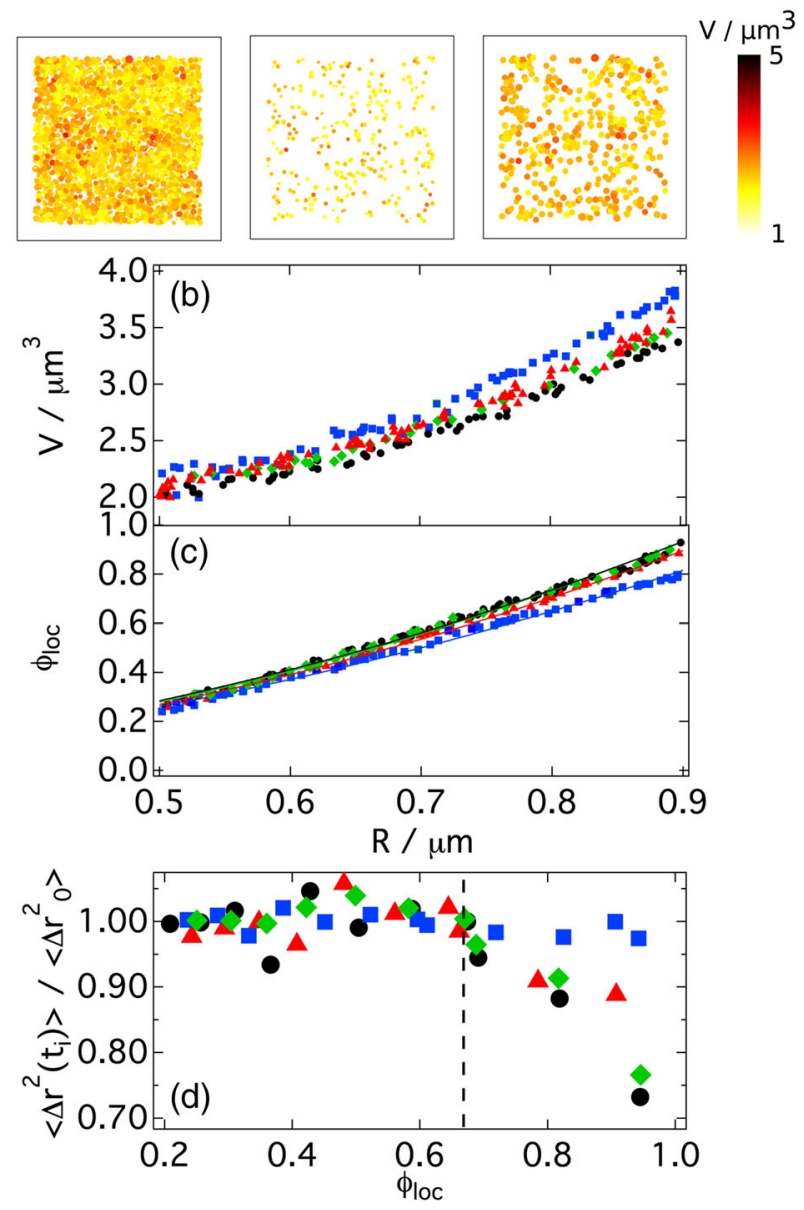

FIG. 4. (a) Projection of a 5 - $\mu \mathrm{m}$-thick and $51 \times 51 \mu \mathrm{m}^{2}$-wide slice of the sample with total volume fraction $\phi=0.61$. All, the $20 \%$ smallest, and the $20 \%$ largest particles are shown (left to right). Particle radius $R$ and Voronoi volume $V$ are represented by the points' sizes and colors (as indicated), respectively. (b) Voronoi volume $V$ and (c) local volume fraction $\phi_{\text {loc }}$ as a function of particle radius $R$. The solid lines represent power-law fits [56]. (d) Normalized squared localization length $\left\langle\Delta r^{2}\left(t_{\mathrm{i}}\right)\right\rangle /\left\langle\Delta r_{0}^{2}\right\rangle$ as a function of $\phi_{\mathrm{loc}}$. The vertical dashed line indicates $\phi_{\mathrm{loc}}=0.67$. Data are shown for total volume fractions $\phi=0.54$ (square), 0.58 (triangle), 0.59 (diamond), and 0.61 (circle).

For each stack, the entire range of particle sizes is divided into 30 groups and for each group the average Voronoi volume calculated. With increasing particle radius $R$, the Voronoi volume $V$ increases for all $\phi$ [Fig. 4(b)]. With increasing $\phi$, the Voronoi volume $V$ becomes smaller and increases slightly less with $R$. For the largest volume fraction, $\phi=0.61$, and the largest particles, the Voronoi volume approaches the particle volume and hence almost no free volume remains.

The ratio between the particle and Voronoi volumes is the local volume fraction of each particle, $\phi_{\text {loc }}=4 \pi R^{3} / 3 \mathrm{~V}$. It covers a broad range, $0.2 \lesssim \phi_{\text {loc }} \lesssim 1.0$, and increases considerably with $R$ [Fig. 4(c)]. For small $R$, the local volume fraction $\phi_{\text {loc }}$ is comparable for all $\phi$. For $R \gtrsim\langle R\rangle$, however, a larger increase of $\phi_{\text {loc }}$ is observed for the larger $\phi$ with $\phi_{\text {loc }}$ approaching 1 for the largest $\phi=0.61$, consistent with the results for $V$.

These findings suggest a link between the localization length $L(R)$ (Fig. 2, inset) and the local volume fraction $\phi_{\text {loc }}(R)$ [Fig. 4(c)] [56]. For the arrested states, $\phi=0.59$ and 0.61 , the localization length $L$ appears to be constant at small $\phi_{\text {loc }}$ but significantly decreases beyond $\phi_{\text {loc }} \approx 0.67$ [Fig. 4(d)]. Similar behavior is found for $\phi=0.58$, where a smaller reduction of the localization length is observed, again, beyond $\phi_{\text {loc }} \approx 0.67$. For $\phi=0.54$, a sample which shows no arrest, no significant drop of the localization length is found. This suggests that localization becomes stronger for $\phi_{\text {loc }} \gtrsim 0.67$ where the absolute value of the localization length and the magnitude of its drop depend on $\phi$, especially on whether the sample is arrested. Thus, local crowding beyond $\phi_{\text {loc }} \approx 0.67$ results in strong localization. This, however, affects only a small fraction of the particles, the largest particles. The dynamical arrest of the whole sample therefore must also rely on structures beyond individual particles and hence involve collective behavior [27].

In conclusion, we investigated concentrated dispersions of polydisperse hard-sphere-like particles. Our results provide the first experimental evidence that the dynamics of the different subpopulations become significantly different beyond the glass transition. Larger particles exhibit significantly slower dynamics with a stronger localization, which coincides with a larger local volume fraction and hence a crowded environment. Beyond a specific local volume fraction $\phi_{\text {loc }} \approx 0.67$, particle localization becomes significantly stronger. This indicates a link between local crowding and dynamical heterogeneities in the glass, and therefore, relates dynamical behavior to structure and ultimately the particle size distribution. Strong localization mainly affects the large particles, which represent a small fraction of the sample only. The small and intermediate particles dominate the average dynamics of glassy samples and hence the glass transition cannot directly be associated with a slowing down of the dynamics beyond a specific value of $\phi_{\text {loc }}$. This suggests that the glass transition also requires collective effects that involve groups of particles. Therefore, the different behaviors of the subpopulations as well as their interplay might need to be considered to fully understand the dynamical slowing down and arrest as well as the glass transition in general. A description based on average behavior hence might not be sufficient. This is expected to also apply to other systems, such as polymer blends [57] or multicomponent metallic glasses [58], and to other topics, such as crystallization [7-14] or random close packing [32-35].

We thank A. B. Schofield (University of Edinburgh) for providing the PMMA particles. This work was supported by the Deutsche Forschungsgemeinschaft (DFG) within the Research Unit FOR1394, project P2, and Grant INST 
208/617-1 FUGG funding the confocal microscope. M. L. acknowledges funding from the Secretaría de Educación Publica (SEP), Programa para el Desarrollo Profesional Docente (PROMEP), through Grant No. SEP-23-005.

[1] W. B. Russel, D. A. Saville, and W. R. Schowalter, Colloidal Dispersions (Cambridge University Press, Cambridge, England, 1989).

[2] D. F. Evans and H. Wennerström, The Colloidal Domain: Where Physics, Chemistry, Biology, and Technology Meet (Wiley-VCH, New York, 1999).

[3] S. Iacopini, T. Palberg, and H. J. Schöpe, Ripeningdominated crystallization in polydisperse hard-sphere-like colloids, Phys. Rev. E 79, 010601 (2009).

[4] E. van den Pol, D. M. E. Thies-Weesie, A. V. Petukhov, G. J. Vroege, and K. Kvashnina, Influence of polydispersity on the phase behavior of colloidal goethite, J. Chem. Phys. 129, 164715 (2008).

[5] D. V. Byelov, M. C. D. Mourad, I. Snigreva, A. Snigrev, A. V. Petukhov, and H. N. W. Lekkerkerker, Experimental observation of fractionated crystallization in polydisperse platelike colloids, Langmuir 26, 6898 (2010).

[6] R. Kurita, D. B. Ruffner, and E. R. Weeks, Measuring the size of individual particles from three-dimensional imaging experiments, Nat. Commun. 3, 1127 (2012).

[7] M. Fasolo and P. Sollich, Fractionation effects in phase equilibria of polydisperse hard-sphere colloids, Phys. Rev. E 70, 041410 (2004).

[8] P. Sollich and N. B. Wilding, Polydispersity induced solidsolid transitions in model colloids, Soft Matter 7, 4472 (2011).

[9] R. P. Sear, Phase separation and crystallisation of polydisperse hard spheres, Europhys. Lett. 44, 531 (1998).

[10] P. N. Pusey, The effect of polydispersity on the crystallization of hard spherical colloids, J. Phys. (Les Ulis, Fr.) 48, 709 (1987).

[11] R. McRae and A. D. J. Haymet, Freezing of polydisperse hard spheres, J. Chem. Phys. 88, 1114 (1988).

[12] P. G. Bolhuis and D. A. Kofke, Monte Carlo study of freezing of polydisperse hard spheres, Phys. Rev. E 54, 634 (1996).

[13] P. Bartlett, A geometrically-based mean-field theory of polydisperse hard-sphere mixtures, J. Chem. Phys. 107, 188 (1997).

[14] J. L. Barrat and J. P. Hansen, On the stability of polydisperse colloidal crystals, J. Phys. (Les Ulis, Fr.) 47, 1547 (1986).

[15] P. J. Lu and D. A. Weitz, Colloidal particles: crystals, glasses, and gels, Annu. Rev. Condens. Matter Phys. 4, 217 (2013).

[16] E. Zaccarelli, C. Valeriani, E. Sanz, W. C. K. Poon, M. E. Cates, and P. N. Pusey, Crystallization of Hard-Sphere Glasses, Phys. Rev. Lett. 103, 135704 (2009).

[17] E. Sanz, C. Valeriani, E. Zaccarelli, W. C. K. Poon, M. E. Cates, and P. N. Pusey, Avalanches mediate crystallization in a hard-sphere glass, Proc. Natl. Acad. Sci. U.S.A. 111, 75 (2014).

[18] C. P. Royall, E. C. M. Vermolen, A. van Blaaderen, and H. Tanaka, Controlling competition between crystallization and glass formation in binary colloids with an external field, J. Phys. Condens. Matter 20, 404225 (2008).

[19] M. Leocmach and H. Tanaka, A novel particle tracking method with individual particle size measurement and its application to ordering in glassy hard sphere colloids, Soft Matter 9, 1447 (2013).

[20] Z. Zhang, P. J. Yunker, P. Habdas, and A. G. Yodh, Cooperative Rearrangement Regions and Dynamical Heterogeneities in Colloidal Glasses with Attractive versus Repulsive Interactions, Phys. Rev. Lett. 107, 208303 (2011).

[21] G. L. Hunter and E. R. Weeks, The physics of the colloidal glass transition, Rep. Prog. Phys. 75, 066501 (2012).

[22] E. R. Weeks, J. C. Crocker, A. C. Levitt, A. Schofield, and D. A. Weitz, Three-dimensional direct imaging of structural relaxations near the colloidal glass transition, Science $\mathbf{2 8 7}$, 627 (2000).

[23] P. N. Pusey and W. van Megen, Phase behaviour of concentrated suspensions of nearly hard colloidal spheres, Nature (London) 320, 340 (1986).

[24] W. K. Kegel and A. van Blaaderen, Direct observation of dynamical heterogeneities in colloidal hard-sphere suspensions, Science 287, 290 (2000).

[25] Z. Brown, M. J. Iwanicki, M. D. Gratale, X. Ma, A. G. Yodh, and P. Habdas, Correlated rearrangements of disordered colloidal suspensions in the vicinity of the reentrant glass transition, Europhys. Lett. 115, 68003 (2016).

[26] A. Latka, Y. Han, A. M. Alsayed, A. B. Schofield, A. G. Yodh, and P. Habdas, Particle dynamics in colloidal suspensions above and below the glass-liquid re-entrance transition, Europhys. Lett. 86, 58001 (2009).

[27] W. Götze, Complex Dynamics of Glass-Forming Liquids (Oxford Science Publications, New York, 2012).

[28] W. van Megen, T. C. Mortensen, S. R. Williams, and J. Müller, Measurement of the self-intermediate scattering function of suspensions of hard spherical particles near the glass transition, Phys. Rev. E 58, 6073 (1998).

[29] G. Brambilla, D. El Masri, M. Pierno, L. Berthier, L. Cipelletti, G. Petekidis, and A. B. Schofield, Probing the Equilibrium Dynamics of Colloidal Hard Spheres above the Mode-Coupling Glass Transition, Phys. Rev. Lett. 104, 169602 (2010); W. van Megen and S. R. Williams, Comment, Phys. Rev. Lett. 104, 169601 (2010); G. Brambilla, D. El Masri, M. Pierno, L. Berthier, L. Cipelletti, G. Petekidis, and A. Schofield, Reply, Phys. Rev. Lett. 104, 169602 (2010); J. Reinhardt, F. Weysser, and M. Fuchs, Comment, Phys. Rev. Lett. 105, 199604 (2010); G. Brambilla, D. El Masri, M. Pierno, L. Berthier, and L. Cipelletti, Reply, Phys. Rev. Lett. 105, 199605 (2010).

[30] E. Zaccarelli, S. M. Liddle, and W. C. K. Poon, On polydispersity and the hard sphere glass transition, Soft Matter 11, 324 (2015).

[31] T. Voigtmann, Dynamics of colloidal glass-forming mixtures, Phys. Rev. E 68, 051401 (2003).

[32] W. Schaertl and H. Silescu, Brownian dynamics of polydisperse colloidal hard spheres: Equilibrium structures and random close packings, J. Stat. Phys. 77, 1007 (1994).

[33] K. W. Desmond and E. R. Weeks, Influence of particle size distribution on random close packing of spheres, Phys. Rev. E 90, 022204 (2014). 
[34] A. Santos, S. B. Yuste, M. Lopez de Haro, G. Odriozola, and V. Ogarko, Simple effective rule to estimate the jamming packing fraction of polydisperse hard spheres, Phys. Rev. E 89, 040302 (2014).

[35] R. Kurita and E. R. Weeks, Experimental study of randomclose-packed colloidal particles, Phys. Rev. E 82, 011403 (2010).

[36] J. Bosse and Y. Kaneko, Self-Diffusion in Supercooled Binary Liquids, Phys. Rev. Lett. 74, 4023 (1995).

[37] A. J. Moreno and J. Colmenero, Anomalous dynamic arrest in a mixture of large and small particles, Phys. Rev. E 74, 021409 (2006).

[38] T. Voigtmann and J. Horbach, Double Transition Scenario for Anomalous Diffusion in Glass-Forming Mixtures, Phys. Rev. Lett. 103, 205901 (2009).

[39] T. Sentjabrskaja, E. Zaccarelli, C. De Michele, F. Sciortino, P. Tartaglia, T. Voigtmann, S. U. Egelhaaf, and M. Laurati, Anomalous dynamics of intruders in a crowded environment of mobile obstacles, Nat. Commun. 7, 11133 (2016).

[40] T. Voigtmann, Multiple glasses in asymmetric binary hard spheres, Europhys. Lett. 96, 36006 (2011).

[41] J. Hendricks, R. Capellmann, A. B. Schofield, S. U. Egelhaaf, and M. Laurati, Different mechanisms for dynamical arrest in largely asymmetric binary mixtures, Phys. Rev. E 91, 032308 (2015).

[42] B. Cabane, J. Li, F. Artzner, R. Botet, C. Labbez, G. Bareigts, M. Sztucki, and L. Goehring, Hiding in the Plain View: Colloidal Self-Assembly from Polydisperse Populations, Phys. Rev. Lett. 116, 208001 (2016).

[43] F. Boulogne, Y. L. Kong, J. K. Nunes, and H. A. Stone, Effect of the Polydispersity of a Colloidal Drop on Drying Induced Stress as Measured by the Buckling of a Floating Sheet, Phys. Rev. Lett. 116, 238001 (2016).

[44] A. Fall, B. Weber, M. Pakpour, N. Lenoir, N. Shahidzadeh, J. Fiscina, C. Wagner, and D. Bonn, Sliding Friction on Wet and Dry Sand, Phys. Rev. Lett. 112, 175502 (2014).

[45] A. G. Mailer, P. S. Clegg, and P. N. Pusey, Particle sizing by dynamic light scattering: non-linear cumulant analysis, J. Phys. Condens. Matter 27, 145102 (2015).

[46] K. L. Planken and H. Cölfen, Analytical ultracentrifugation of colloids, Nanoscale 2, 1849 (2010).
[47] J. C. Crocker and D. G. Grier, Methods of digital video microscopy for colloidal studies, J. Colloid Interface Sci. 179, 298 (1996).

[48] A. D. Dinsmore, E. R. Weeks, V. Prasad, A. C. Levitt, and D. A. Weitz, Three-dimensional confocal microscopy of colloids, Appl. Opt. 40, 4152 (2001).

[49] V. Prasad, D. Semwogerere, and E. R. Weeks, Confocal microscopy of colloids, J. Phys. Condens. Matter 19, 113102 (2007).

[50] J. C. Conrad, F. W. Starr, and D. A. Weitz, Weak correlations between local density and dynamics near the glass transition, J. Phys. Chem. B 109, 21235 (2005).

[51] C. P. Royall, W. C. K. Poon, and E. R. Weeks, In search of colloidal hard spheres, Soft Matter 9, 17 (2013).

[52] M. C. Jenkins and S. U. Egelhaaf, Confocal microscopy of colloidal particles: Towards reliable, optimum coordinates, Adv. Colloid Interface Sci. 136, 65 (2008).

[53] The restriction to one slice results in a rapid data acquisition and hence access to the short-time dynamics and, furthermore, a reduced data volume and hence access to the longtime dynamics.

[54] The quoted particle radii are averages over the 20 stacks obtained during steps (i) and (iii) if the particles remain in the observation volume, or averages over the 10 stacks obtained during steps (i) or (iii) if the particles leave or enter the observation volume during the experiment, respectively. The sizes of particles that appear and disappear in sequence (ii) cannot be determined and hence these particles are neglected. Because of their short appearance, they only contribute to the short-time dynamics which anyway have good statistics and are not the focus of this study.

[55] C. H. Rycroft, Voro++: A three-dimensional Voronoi cell library in C++, Chaos 19, 041111 (2009).

[56] We assume that the dependence of $\phi_{\text {loc }}$ on $R$ can be described by the empirical relation $\phi_{\text {loc }}=A(R /\langle R\rangle)^{\alpha}$. Fits yield $A=0.543,0.583,0.607,0.609$ and $\alpha=1.94$, $2.05,2.06$, and 2.02 for $\phi=0.54,0.58,0.59$, and 0.61 , respectively.

[57] K. Binder, J. Baschnagel, and W. Paul, Glass transition of polymer melts: test of theoretical concepts by computer simulations, Prog. Polym. Sci. 28, 115 (2003).

[58] W. H. Wang, C. Dong, and C. H. Shek, Bulk metallic glasses, Mater. Sci. Eng. R 44, 45 (2004). 